\title{
Coupling occupant behaviour with a building energy model - A FMI application
}

\author{
Gilles Plessis $^{1} \quad$ Édouard Amouroux ${ }^{2} \quad$ Yvon Haradji $^{1}$ \\ ${ }^{1}$ EnerBaT - EDF R\&D, Moret sur Loing, FRANCE \\ ${ }^{2}$ LIP6 - Université de Paris 6, Paris, FRANCE \\ gilles.plessis@edf.fr_ edamouroux@gmail.com_yvon.haradji@edf.fr
}

\begin{abstract}
This paper illustrates the use of Functional Mockup Interface (FMI) [1] to couple an occupant behaviour simulator and a building model.

Due to their intrinsic nature, occupant behaviour and building and its energy systems are usually represented by different modelling paradigms. The occupant behaviour is here described by Agent-Based Modelling (ABM) whereas the building is described by a set of hybrid and differential algebraic equations, typical of dynamic thermal modelling. Such different complex systems cannot be efficiently simulated in a single tool. Therefore, one solution is the tool coupling approach.

The FMI standard for co-simulation was used to couple the SMACH occupant behaviour simulator and a building energy model built with the BuildSysPro Modelica library. Variables of interest are passed from one model to another at fixed synchronization time steps.
\end{abstract}

Keywords: Building simulation; behavioural modelling; Specific use of electricity; thermal comfort; Modelica; FMI; co-simulation

\section{Introduction}

Enforcement of energy efficiency policies drives new buildings towards better performance and especially low or even positive energy buildings. These kinds of buildings are different from existing ones as their ventilation and envelope heat losses are intensively decreased. Nevertheless, this improvement has two consequences. First, human actions to ensure thermal comfort can cut back heating and cooling energy savings. Second, a significant share of energy will be consumed by specific electricity uses (light- ing, cooking, white goods, electronic appliances...) and not anymore by space heating and cooling. Both points are strongly linked with occupant behaviour.

Occupant behaviour is commonly described in dynamic building simulation tools using standardized occupancy profiles. Various studies suggest that occupant behaviour should be taken into account in a more accurate way, as it can have a dramatic impact on energy consumption especially in the context of low and positive energy buildings [2] [3].

The purpose of this work is to couple realistic occupant behaviours with building energy simulation. It focuses on dynamic modelling and especially electric power demand instead of energy consumption. Two interaction approaches were carried out:

- Co-simulation for R\&D studies,

- Generation of realistic occupancy scenarios for simplified building simulation tools.

This last objective will be fulfilled thanks to design of experiments using the co-simulation. Only the tool-coupling approach is presented here.

\section{Occupant behaviour model}

The occupant behaviour model is implemented in the SMACH platform, an agent-based tool developed by EDF and the LIP6 laboratory.

The coupling between the building energy model and the occupant behaviour model is mainly carried out through thermal comfort perception and control of electrical appliances.

\subsection{Occupant behaviour model}

In order to represent adequately and individually the behaviour of each occupant, we rely on an agentbased modelling approach and, more specifically, the 
one we already applied in [4]. The agents, in SMACH, provide a realistic way of modelling inhabitants' behaviour. In the occupant behaviour model, an agent corresponds to an individual person, and a family corresponds to a group of agents. This modelling approach emphasis the fact that agents are autonomous and have their own individual interaction dynamic within the situation. At each moment, their universe is defined by their individual perception of the situation, their comfort level and their preferences regarding possible actions [5]. Stategraphs could have been used however this kind of approach is much less adaptable and concise than $\mathrm{ABM}$ for occupant behaviour modelling.

In practice, the inhabitants' activity is decomposed into generic tasks (t) such as "watch TV" or "cook dinner". The actual behaviour of each individual is a set of actions (a) derived from the generic tasks. A task is a tuple $\left(\tau_{\min }, \tau_{\max }, \mathrm{E}_{\mathrm{t}}, \mathrm{E}_{\mathrm{ff}}, \mathrm{T}_{\text {pre }}\right)$ corresponding respectively to minimal and maximal duration to conduct the task, the required and favourable appliances and the pre-conditional tasks. For instance, ironing may require the completion of cleaning clothes, lasts one to two hours, requires the iron appliance and may be likely to be conducted with the TV switched on.

An action is an instantiation of a task by an occupant. It is defined by a tuple $a=(t, w, s t)$ corresponding respectively to the associated task, rhythm and its state (done, not-done). A rhythm (w) is a tuple (per, freq, var, $P P)$ corresponding respectively to the base period (day, week, month, year), the frequency, the frequency variability and a set of preferred periods (PP). An action example could be as follows: a child may watch TV according to a weekly rhythm of 10 viewing periods on weekdays between 7 and 8 a.m and/or 5 and 6 p.m.

\subsection{Thermal comfort model}

The thermal comfort model used in the occupant behaviour simulator is derived from Fanger's PMV model [6]. Instead of defining a mean comfort value, an individual thermal comfort level is defined after the same set of variables (air temperature, radiative temperature, humidity, metabolic heat production and mechanical work). A new parameter called frilosity defined by expert assessment and based on field studies in real situation is also taking into account. It describes the cold tolerance of each occupant [7]. On top of this individual model, a group comfort level is defined per room as follows:

$$
G C=\sum_{i \in n} \frac{\operatorname{comfort}_{i} \cdot \text { age }_{i}}{n}
$$

Where $a g e_{i}$ is the age class and comfort $_{i}$ the individuals comfort. The age class defines a level of responsibility depending on age, for instance an adult will favour a child comfort instead of his/her. This group comfort level is used to determine what action the group will choose (e.g. increase temperature set point, open windows) and individuals' actions (e.g. adapt clothing, change activity ...).

\subsection{Appliances}

Electrical appliances, $e \in E$, are defined by their electrical power consumption $\theta_{i}$. The power function defines this relation for each appliance.

$$
\text { power : }\left\{\begin{array}{cc}
E & \rightarrow \mathbb{R}^{+} \\
e & \mapsto \theta_{i}
\end{array}\right.
$$

We consider two types of electrical appliance: state-based appliances and program-based appliances.

State-based appliances are defined as a tuple $\left(\theta_{o}\right.$, $\left.\theta_{s}, s t\right)$ where $\theta_{o}$ and $\theta_{s}$ are the electrical power consumptions when $e$ is running or in standby mode, and st $\in$ \{off, standby, on $\}$ is the state of the appliance, modified by occupants in the house during their activities. Heaters, TVs, fridges... can be represented by state-based appliances.

Program-based appliances are defined as an ordered pair $\left(P_{e}, s t\right)$ where $P_{e}$ is a set of operating programs characterised by load curves. The status $s t$ of the appliance is then defined by an ordered pair $s t$ $=(p, t)$ where $p$ is the currently selected program and $t$ is the time since the beginning of this program. A program $p$ is an ordered pair $(\tau, \varphi)$ where $\tau$ is the program duration and $\varphi:[0, \tau] \rightarrow \mathbb{R}^{+}$gives the appliances power consumption over time during the program. Thus, power $(e)=\varphi_{\mathrm{p}}(t)$. For instance, washing machines can be represented by this kind of appliance.

In SMACH, all energy consumption profiles come from real data from the REMODECE European project [8].

\section{Building energy model}

The building energy model is written in Modelica language with the BuildSysPro library developed by EDF [9]. We used a purely thermal model compliant with the Thermal.HeatTransfer class from the Modelica standard library. The class is defined by its connector involving the temperature $T$ as a potential and $Q \_$flow for the heat flow rate. 


\subsection{Building envelope}

The "Mozart" house is one of the most representative houses in the French residential building stock, and was therefore chosen for this work. It is a medium size detached house of $100 \mathrm{~m}^{2}$ of living surface area and an air volume of $252.15 \mathrm{~m}^{3}$.

The building is modelled in a low energy configuration: the U-values of the different envelope components are low compared to the standard French building stock. Internal wall insulation is chosen since this is the most common in France; it impacts the potential of heat storage into walls. The internal walls are modelled in the same way as the other opaque walls. Therefore, they contribute to the thermal inertia.

Table 1: Main parameters of the building envelope

\begin{tabular}{|l|r|c|}
\hline PARAMETER & VALUE & UNIT \\
\hline $\mathrm{U}_{\text {outdoor wall }}$ & 0.27 & {$\left[\mathrm{~W} \cdot \mathrm{m}^{-2} \cdot \mathrm{K}^{-1}\right]$} \\
$\mathrm{U}_{\text {ceiling }}$ & 0.191 & {$\left[\mathrm{~W} \cdot \mathrm{m}^{-2} \cdot \mathrm{K}^{-1}\right]$} \\
$\mathrm{U}_{\text {floor }}$ & 0.263 & {$\left[\mathrm{~W} \cdot \mathrm{m}^{-2} \cdot \mathrm{K}^{-1}\right]$} \\
$\mathrm{U}_{\text {windows }}$ & 1.43 & {$\left[\mathrm{~W} \cdot \mathrm{m}^{-2} \cdot \mathrm{K}^{-1}\right]$} \\
\hline
\end{tabular}

Windows are double-glazed, with no thermal inertia. The conductive, convective and radiative heat transfers are considered.

The building envelope model is composed of six zones corresponding to the different rooms represented in Figure 1. The garage is not taken into account.

\subsection{Boundary conditions}

Weather data is applied as boundary conditions on the outdoor side of the building model. The building envelope is studied in a temperate climate, more precisely the weather data from Trappes, a city lo- cated near Paris in France. The weather reader model provides the outdoor dry air temperature, the direct and diffuse solar radiations and the sky temperature. Relative humidity and wind data (velocity and direction) are not used in this first study.

The short wave radiations transmitted inside the building envelope through the windows are entirely absorbed by the floor. The long wave radiative heat transfers which occur between the sky and surroundings and the external surfaces of the walls and windows are taken into account through a combined heat transfer coefficient.

\subsection{Model inputs}

Internal heat gains due to the occupants are set to $90 \mathrm{~W} \cdot \mathrm{pers}^{-1}$. The indoor occupancy scenario is coupled to the occupant behaviour model, impacting internal gains and set point temperatures, which are specified in each zone by the occupants depending on their thermal comfort perception. Occupants also have the possibility to open windows if needed to ensure their comfort. The window open/closed status has an impact on the internal gains by adding a heat gain or loss, depending on the external weather conditions.

\subsection{HVAC system}

The HVAC system is composed of an ideal electrical heater controlled by PID and a mechanical ventilation. A static model is used for the ventilation system with a fixed air change rate.

Considering the weather conditions of Trappes and a normative scenario for occupancy specifying internal heat gains, the annual heat demand for this building is $20 \mathrm{kWh} \cdot \mathrm{m}^{-2} \cdot$ year $^{-1}$.

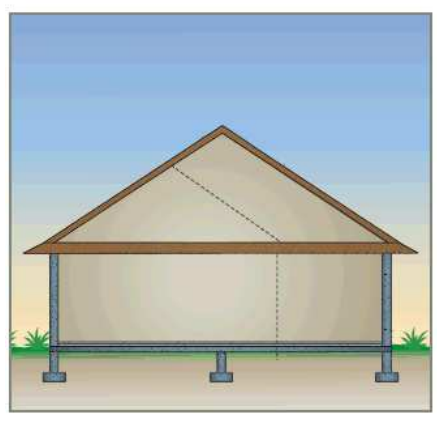

Mozart

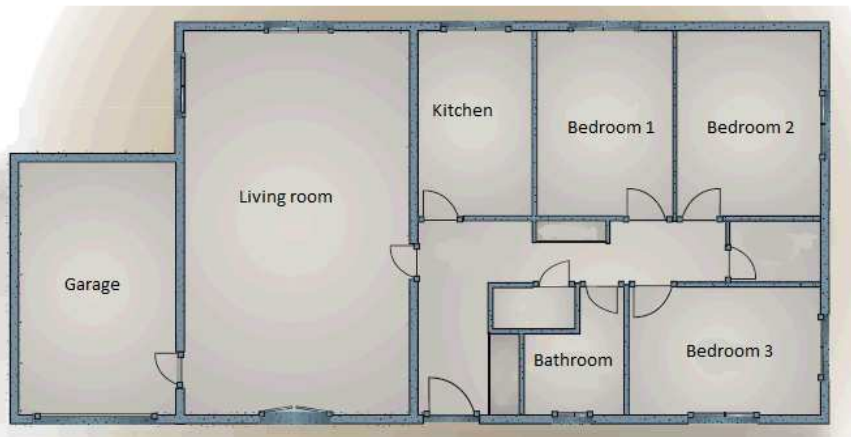

RDC

Figure 1: Diagrams of the Mozart house 
Figure 2 shows the overall Modelica model.

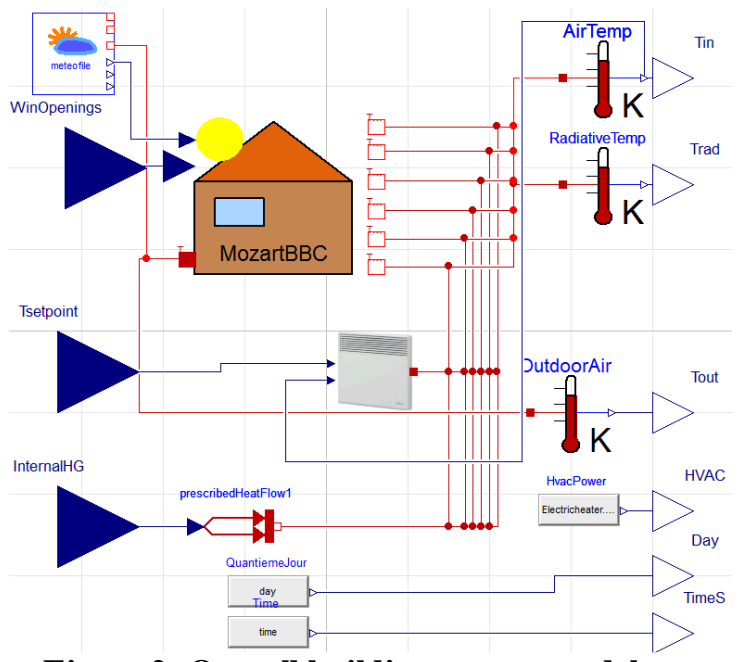

Figure 2: Overall building energy model

\section{Co-simulation}

As previously mentioned, the model describing the building and its energy system has been developed using the BuildSysPro library with Dymola 2014. The occupant model has been implemented within the SMACH agent-based simulation platform, written in Java. In order to allow co-simulation of heterogeneous simulators, the Modelica building model was exported from Dymola as a Functional Mock-up Unit (FMU), based on the Functional Mock-up Interface (FMI) for co-simulation standard. This approach has already been applied in several works [10] [11].

\subsection{Implementation}

FMI defines a standardized interface to be used in computer simulations to develop complex systems [1]. In practice, it defines a common interface to control the FMU as a dynamic library, a .dll file in the present case. The first version of the standard was used.

The master algorithm part is integrated into the SMACH occupant simulator. The JFMI wrapper [12] was used to control the exported FMU within the SMACH platform. It is designed to facilitate control of a native library with Java. A centralised architecture was used with a single FMU representing the entire building with its energy systems. The SUNDIALS CVODE solver with backward differentiation formula [13] was integrated as the built-in solver of the FMU.

\subsection{Coupling variables}

Several coupling variables are selected in order to couple the building energy model and the occupant simulator. On one hand, the occupant simulator must supply data that can impact the thermal ambiance of each room in the building or the HVAC system. On the other hand, the building energy model must return information to estimate the overall power load and the thermal comfort of the occupants. Therefore the FMU inputs are:

- The temperature set point of each room where occupants can set the temperature.

- The internal heat gains due to appliances and occupancy.

- The windows opening status.

The FMU outputs are:

- The air temperature in each room.

- The mean radiative temperature in each room.

- Outdoor temperature.

- Electrical power consumption of the HVAC system

There are also two optional outputs to ensure proper synchronisation:

- Day

- Time in second

\subsection{Workflow}

The master algorithm acts as a scheduler between the FMU and the occupant model within the SMACH platform. We use a constant synchronization time step of 1 minute. After the instantiation and initialisation steps, the workflow is the following:

1. The behaviour of each occupant is computed based on his/her perceptions (action of other individuals, temperature of the room...) and internal state (action that he yet has to perform, thermal comfort level...).

2. The FMU inputs corresponding to occupants' actions on thermal environment are set (heater control, opening of the windows...).

3. The building energy model computes the actual temperatures based on the occupants actions and their presence (internal heat gains)

4. The FMU outputs are returned to the occupant simulator.

\subsection{Computing performances}

On a computing time point of view, the coupling is heavy. For a one month simulation and a time step of 1 minute, the occupant behaviour simulation takes 3 minutes with SMACH and the building energy simulation takes 10 seconds with Dymola. When coupling 
is applied, the same simulation takes 13 minutes. These computing times have been obtained with standard laptop equipped with an Intel i5 $2520 \mathrm{M}$ processor on Windows 732 bits. These are rough results and no optimisation has been conducted yet.

\section{Results}

This section shows some outputs from the cosimulation between SMACH and the BuildSysPro building model.

The activity diagram in SMACH, Figure 3, is used to analyse simulations. Activities are represented by different colours for each occupant over time.

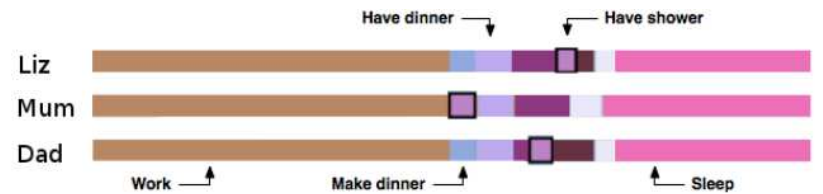

Figure 3: Activity diagram, a working day pattern

On Figure 4, the activity diagram clearly shows the difference between usual working days and other days (Wednesday and weekend) for this household.

Figure 5 shows the temperature evolution over thirteen days including seven days of vacation. The blue line represents the outdoor temperature and the red line, the indoor temperature in the living room. The grey stripes show when the HVAC system is working.

In fact, the actual temperatures and power consumption curves differ because during regular periods, the family is not at home during weekday daytime and sets the objective to $18^{\circ} \mathrm{C}$ whereas the indoor temperature is set according to each individual/group comfort level when they are at home. On the contrary, during holidays the indoor temperature is set at $12^{\circ} \mathrm{C}$ thus, the electric heater power load is null for an extended period of time (due to the efficiency of the represented low energy house) and then can oscillate to maintain this temperature. One may also observe the difference between weekdays and weekends. The absence of occupants during weekdays lets the HVAC system controls the temperature according to the temperature set points.

\section{Conclusion}

This paper presents the first results of the cosimulation between the SMACH platform for occupant behaviour and a BuildSysPro building model. The tool coupling is fully functional, however to ensure computer time efficiency and adaptability the following improvements will be considered in IEA Annex 60 [14]:

- Control of the communication step size,

- Dividing the Modelica model into sub-models (HVAC system, building envelope) and use composition of FMUs to ensure adaptability,

- Auto-mapping of coupling variables, considering input and output names and dimensions,

The authors would like to thank the ANR for funding the SUPERBAT project.

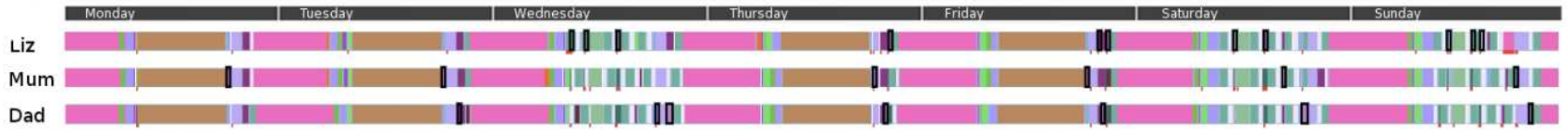

Figure 4: Activity diagram over one week 


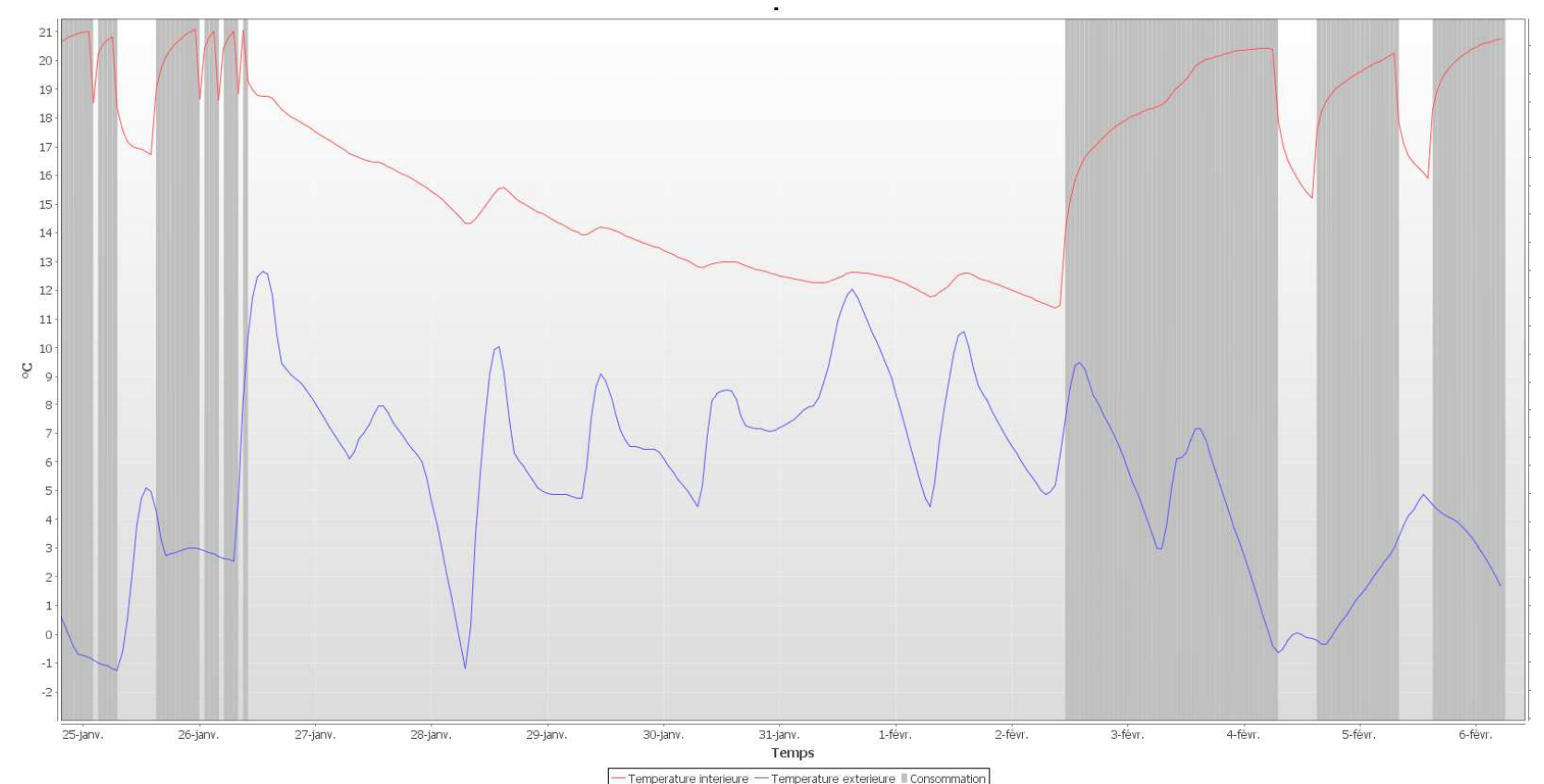

Figure 5: Indoor and Outdoor temperature evolution over holiday period

\section{References}

[1] MODELISAR - ITEA2, Functional Mockup Interface for Co-Simulation, October 2010.

[2] Kashif, A., Ploix, S., Dugdale, J., Le, X.H.B., Simulating the dynamics of occupant behaviour for power management in residential buildings. Energy and Buildings Vol. 56 (2013) p85-93.

[3] Bourgeois, D., Reinhart, C., Macdonal, I., Adding advanced behavioural models in whole building energy simulation: A study on the total energy impact of manual and automated lighting control. Energy and Buildings Vol. 38.

[4] Amouroux, E., Huraux, T., Sempe, F., Sabouret, N., Haradji, Y., Simulating human activities to investigate household energy consumption. Proceedings of the ICAART 2013.

[5] Haradji, Y., Poizat, G., Sempe, F., Human activity and social simulation. Advances in applied human modeling and simulation, $\mathrm{p}$ 416-425, 2012

[6] Fanger, P.O., Thermal comfort: Analysis and applications in environmental engineering. Danish Technical Press, 1970.

[7] Parsons, K. C., The effects of gender, acclimation state, the opportunity to adjust clothing and physical disability on requirements for thermal comfort. Energy \& Buildings, vol. 34, no. 6, pp. 593-599, 2002.

[8] REMODECE consortium, REMODECE deliverables. Can be found at http://remodece.isr.uc.pt/

[9] Plessis, G., Kaemmerlen, Lindsay, A., BuildSysPro: a Modelica library for modelling buildings and energy systems. Proceedings of the International Modelica Conference 2014.

[10] Pazold, M., Burhenne, S., Radon, J., Herkel, S., Antretter, F., Integration of Modelica models into an existing simulation software using FMI for Co-Simulation. Proceedings of the International Modelica Conference 2012.

[11] Viel A., Strong coupling of Modelica system-level models with detailed CFD models for transient simulation of hydraulic components in their surrounding environment. Proceedings of the International Modelica Conference 2011.

[12] Ptolemy consortium, Ptolemy project at http://ptolemy.eecs.berkeley.edu/java/jfmi/

[13] Hindmarsh, A. C., Brown P. N., Grant, K. E., Lee S. L., Serban, R., Shumaker, D. E., Woodward, C. S., SUNDIALS: Suite of nonlinear and differential/algebraic equation solvers. ACM Transactions on Mathematical Software, Vol. 31(3), p. 363-396, 2005.

[14] IEA Annex 60 consortium. Project webpage on http://www.iea-annex60.org/ 